\title{
Topical Metrology Problems in the Era of Cyber-physical Systems and Internet of Things
}

\author{
Roald Taymanov ${ }^{1}$, Kseniia Sapozhnikova ${ }^{1, *}$, and Anton Ionov ${ }^{2}$ \\ ${ }^{1}$ D.I.Mendeleyev Institute for Metrology, 190005 St.Petersburg, 19 Moskovsky pr., Russia \\ ${ }^{2}$ Omsk State Technical University, 644050 Omsk, 11 Mira av., Russia
}

\begin{abstract}
The paper deals with metrological tasks conditioned by the coming technological revolution that is connected with the application of cyber-physical systems (CPSs) and Internet of Things (IoT). The need for terminology standardization in this field is shown and definitions of the main terms are proposed. CPSs and IoT can be economically efficient only in case of the transition to a qualitatively new level of metrological maintenance in the course of operation. It is necessary to abandon conventional methods of periodic calibrations. Topical metrology problems with regard to multi-channel systems including the necessity to check the reliability of measurement results automatically, are formulated. A number of examples demonstrate that to solve such problems it is possible to rely on the experience gained in the development of measuring systems with the metrological self-check and on the first national documentary standards in this field.
\end{abstract}

\section{Introduction}

In recent years, scientific and technical literature has often focused on cyber-physical systems (CPSs) and Internet of Things (IoT) that "will bring advances in personalized health care, emergency response, traffic flow management, and electric power generation and delivery, as well as in many other areas now just being envisioned" [1].

Such projects as "smart manufacturing", "smart energetics", "smart transport", "smart healthcare", "safe smart city", etc., are at the stage of development and, in some cases, at the stage of experimental operation. Wide and prompt introduction of the projects of such a type is inevitable and predetermined by economic reasons, but if their metrological challenges are underestimated, it can be dangerous.

\section{Specific problems of metrology}

\subsection{Problems of terminology}

CPSs and the IoT are not something fundamentally new. They characterize a new stage in the development of technologies. Different companies develop various systems and devices depending on their own plans. Therefore, the transition to CPSs and IoT is decentralized [2-4].

The decentralized development, in its turn, leads to the emergence of a large number of terms offered by various authors, which describe concepts in this field, e.g., Internet of Things, Cyber-Physical Systems, Internet of Everything, Industrial Internet, Pervasive
Computing, Pervasive Sensing, Ubiquitous Computing, Wireless Sensor Networks, Smart Objects, Cooperating Objects, Machine-to-Machine, Ambient Intelligence, and Operational Technology / Information Technology convergence [3], Smart Grid, World Wide Sensor Net, Smart Building Environment, etc.

Most of people consider basic terms "Cyber-Physical System" and "Internet of Things" to be synonyms. However, the analysis of literature shows that although they are close to each other, significant differences exist.

Practically, all the CFC definitions include integration of calculation algorithms with physical components [1, 4-9]. For example, CPSs are:

- "engineered systems that are built from, and depend upon, the seamless integration of computational algorithms and physical components" [7];

- systems "of collaborating computational elements controlling physical entities" [4];

- "co-engineered interacting networks of physical and computational components [1];

- "complex, multi-disciplinary, physically-aware next generation engineered systems that integrate embedded computing technology (cyber part) into the physical phenomena by using transformative research approaches" [5];

- "systems consisted of various natural objects, artificial sub-systems, and controllers, enabling forming integrated whole from such a set". Human can be either outside the CPS (human out of loop) or inside it ((human in the loop) [9]:

From the context of the publications mentioned it is clear that they imply large systems solving a certain technical or social task.

\footnotetext{
Corresponding author: k.v.s@,vniim.ru
} 
In the IoT definitions the authors usually emphasize the availability of "things" identifiers and their connections with the Internet $[1,4,10,11-17]$, e.g.:

- "a global infrastructure for the information society, enabling advanced services by interconnecting (physical and virtual) things based on existing and evolving interoperable information and communication technologies. The term "thing" is treated here in the broadest sense of the word. The "thing" can be material (physical) or immaterial (virtual), it can be an object (for example, a production) or a product, animate and inanimate, but it should be necessarily "capable of being identified in time and space and integrated into communication networks" $[10,11]$;

- "a network that connects uniquely identifiable "Things" to the Internet. The "Things" have sensing/actuation and potential programmability capabilities. Through the exploitation of unique identification and sensing, information about the "Thing" can be collected and the state of the 'Thing' can be changed from anywhere, anytime, by anything" [4].

- "a dynamic global network with self-configuring capabilities based on standard and interoperable communication protocols where physical and virtual "things" have identities, physical attributes, and virtual personalities and use intelligent interfaces, and are seamlessly integrated into the information network" [14].

- "a framework in which all things have a representation and a presence in the Internet...." [16].

- "a network of physical objects ("things") that are provided with unique identifiers, which makes it possible to transfer data over a network without human-to-human interaction" [13].

In accordance with [4], "a CPS starts from the interconnection and collaboration of objects in an intranet scenario and can grow to the level of interconnecting objects over the Internet...", while "IoT that starts from the lowest level of identifying an object to read ... data on RFID tags and can grow to the level of networking ... to do collaborative work where, in this case, it grows to the level of CPS. All in all, "IoT targets a broader view of connecting objects in a global aspect" whereas CPS is aimed "at coordinating networked objects to achieve a specific goal".

The authors of the present paper propose the following definitions of two basic terms:

- cyber-physical system is a network of interacting physical and computational components solving certain technical or social tasks. Such systems can be open (with the access to any number of unspecified people), close, and semi-open;

- Internet of Things is a technology that enables connecting uniquely identifiable "things" with the Internet, whereas the state of the 'Thing' can be changed from anywhere, anytime, by any means.

The current situation demonstrates that there is a need for normative support of works in the field of CPSs and IoT, including the development of a specialized terminological dictionary accepted by all interested sides $[13,18]$. The dictionaries will allow engineers, who develop equipment in this field, and consumers to understand each other.

\subsection{The issue of measurement information reliability in CPSs and IoT}

From the position of metrology, the CPSs have a number of features that distinguish them from conventional multichannel measuring systems widely used at present. These features, in the first place, are as follows:

- The number of measuring channels in one CPS even in the coming years can range from hundreds to many hundreds of thousands of units.

- Measuring channels can include sensors measuring various quantities, both electrical and non-electrical, and they can be spaced far apart.

- In many cases, operating conditions of sensors are harsh, the environment being not sufficiently strictly determined.

- During operation, such CPSs can develop, and the number of measuring channels and the variety of measurands can grow.

- In such systems, as a rule, "cloud technologies" are involved.

- Measurement information is transmitted over long distances via wired or wireless channels.

- Measurement results should be transmitted according to standardized protocols, the electronic exchange of measurement data being consistent with SI units and GUM [13].

- Real time processing of data should be carried out.

- Access to sensors for their maintenance, including metrological maintenance, is impeded in many cases.

The listed features of the CPSs pose new tasks for metrology specialists. Without solving them, the coming era of cyber-physical systems and Internet of Things will bring more dangers that hopes.

Analysis of papers and reports on CFSs and IoT shows that, as a rule, the authors of publications emphasize their attention on the algorithms of processing measurement information (or, more accurately, on a virtual model of the object / situation, which is realized using the algorithms in question) [19-21].

At the same time, the idea of minimizing human intervention in the process of collecting information is implicitly postulated, since the number of channels in the CFSs increases so much that their maintenance becomes unrealistic [2, 19, 22].

However, the most part of information which is processed in the CFSs is measurement information and comes from sensors.

Measurement information is a kind of product having a cost and consumer properties that determine this cost. The most important of them are the accuracy, characterized by an attributed uncertainty, and metrological reliability.

The metrological reliability of information is estimated by the probability with which the measurement data coming for further processing during a calibration (verification) interval can be considered reliable. 
At the same time, measurement information is recognized to be reliable if its uncertainty lies within the limits established in the documentation for a measurement procedure and / or an appropriate measuring instrument, including a sensor.

In section 1 it is noted that in the CFSs the operating conditions of the sensors cannot be determined quite strictly. During the calibration interval, changes in measurement conditions may take place, which go beyond the limits specified for operating conditions. Besides, the parameters of the measuring instrument may change in comparison with those stated during the calibration. These changes may occur due to:

- deviation of the metrological characteristics of the sensor used or of those of the measuring channel as a whole, for example, due to ageing;

- changes of environment parameters in the area between the sensor and the measurement object, for example, changes of humidity;

- deviation of parameters of the object itself, e.g., the deviation of its magnetic properties;

- a set of such deviations.

As a result, measurement information coming from a measuring instrument will become unreliable. This circumstance may create the risk of a manufacturing defect, accident, or even crash.

According to some estimations, at present the causes of consequences of such a type are metrological (gradual) failures in $60 \%$ of cases. In practice, however, attempts to avoid such failures using the traditional methods of calibration or verification (even for 2-3 year calibration or verification intervals) are not a key to success. Up to $12 \%$ of measuring instruments coming for regular calibration in the course of their operation, have the uncertainty that exceeds permissible limits.

Therefore, it is clear that the concept of the "metrological reliability of measurement information" is broader than the concept of the "metrological reliability" of a measuring instrument, which according to [23], is defined "as the reliability of a measurement instrument in terms of maintaining its metrological "health". Measurement information can be unreliable even in case if it has been obtained using a measuring instrument that is "healthy" in a metrological sense.

Even simple automation of equipment control, reducing the amount of process control carried out by an operator, increases the requirements for metrological reliability of measurement information used.

It becomes less and less acceptable:

- to check the metrological "health" of measuring instruments by conventional methods of periodic calibration or verification;

- to enhance the reliability of measurement results by shortening calibration (verification) intervals.

In addition, the need arises more and more frequently not to interrupt the operation process of sensors embedded in equipment during 10-15 years due to safety requirements. Therefore, investigations have originated with the aim to search simplified methods of metrological health checking [24].
Creation of CPSs and transition to the IoT implies a sharp increase of the intervals between the maintenance of built-in measuring instruments and the means for processing measurement information. This fact incommensurably increases the requirements for the metrological reliability of measurement information, and actualizes the development of methods and means that ensure their implementation. Metrological failures in large systems can lead to very serious consequences.

However, engineers who are developing the equipment, often believe that the reliability of measurement information is guaranteed by sensor manufacturers, and an additional role of filtering and rejecting measurement results can be performed by the data processing algorithms $[2,20,25,26]$.

Actually, qualitatively new methods for checking the reliability of measurement information are necessary here. New concept of metrological maintenance should take into account fast decrease in cost and acceleration of moral ageing of sensors and other measuring instruments.

Since the expected lifetime of many CPSs will be equal to decades, their modernization should be performed without stopping their operation. The periodic calibration procedures in the course of the operation process should be excluded, as a rule. Instead, gradual replacement of outdated components with newer ones calibrated should be done.

Such an approach stipulates tightening up the requirements for the metrological reliability of CPS components. Fulfillment of these requirements should be provided based on technology, manufacturing culture, and results of accelerated tests. Such tests are applied in metrology practice.

Documentary standards are known, in particular, [27], aimed at metrological comparability of test results. The data on the metrological reliability of sensors intended for operation in the CPS should be included in specifications as mandatory characteristics.

This action will enable shifting towards the ensurance of objects facing with danger due to CPS failures that can lead to significant economic losses. As a result, the responsibility of engineers developing CPSs and IoT and manufacturers will increase.

The steps aimed at enhancing the metrological reliability of sensors and other network components are very important.

However, checking the reliability of measurement information used continued to be a challenge. The metrological failure of a system component is not the only cause for defects in system operation.

To solution of the task by an economically efficient way can be found based on special features of CPSs and other IoT systems as the objects of metrological maintenance.

\subsection{Features of CPSs and other loT systems as the objects of metrological maintenance}

In different CFSs and other components built using the IoT technology, huge amounts of data are generated, 
transmitted and received, including those data that characterize the quality of functioning.

As it was explained above, if measurement information is unreliable (inaccurate), this situation may be dangerous. However, analysis of human bio-sensory systems testifies that development of systems characterized by reliable measurement information is possible. The main features of such systems are:

- predicting the future state by automatic revealing of defects at an early stage of their formation (by the feeling of pain, understanding that sensitivity is worsening or perception is inadequate, etc,);

- sensor redundancy;

- application of compensating information from other sensor systems or supplementary means.

If one relies upon the Nature experience, it is possible to conclude that to ensure the reliability of measurement information, it becomes necessary to predict the metrological state of such systems and, if necessary, to correct measurement results.

In other words, the task of predicting the state of sensors, measuring channels as the whole and measurement objects becomes urgent. Then, on the basis of results obtained, the task arises to develop some correction methods that will allow prolonging the process of functioning a CPS and components of the IoT.

Galar in [28] singles out 4 consequent steps of scientific solution of such a task:

- descriptive analytics (what happened?);

- diagnostic analytics (why did it happened?)

- predictive analytics (what will happen?)

- prescriptive analytics (how can we make it happen?).

With regard to sensors and measuring channels, realization of these steps should stipulate:

- development of methods and, if necessary, embedded measuring instruments providing detection of the growth of uncertainty of sensors and measuring channels;

- determination of the uncertainty growth causes;

- estimation of the term when measurement uncertainty will exceed permissible limits (with high probability) stated in specifications;

- analysis of opportunities and necessity for measurement result correction:

$\checkmark \quad$ if correction is necessary and possible, development of correction algorithms;

$\checkmark \quad$ if correction is impossible, in particular, due to economic reasons, development of methods and instruments informing customers about a coming metrological failure.

For sensors, CPS measuring channels and systems (components) of IoT in the course of their operation process, all the above procedures should be made automatically.

The main difficulty in solving these tasks is connected with detecting the increase in measurement information uncertainty. It is necessary to have reference values with which it is possible to compare the values of corresponding quantities obtained after processing the signals coming from sensors and measuring channels. As a result of such a comparison, to reveal an undesirable effect is possible. It should be noted that the application of a reference standard, as a rule, is excluded since keeping a comparable cost, to provide a higher metrological reliability for a complicated reference standard seems to be unreachable.

However, CPS development was preceded by numerous developments of measuring systems of responsible designation. Usually, they had less measuring channels than CPSs, but like CPSs they were characterized by difficult access to measuring channels and sensors which prevented metrology specialists from performing calibrations (verifications) during many years.

The experience obtained in carrying out these works has demonstrated that under certain conditions for the considered goal, reference values of special quantities formed on the basis of redundancy (applied to a special situation) in a CPS or other systems based on the IoT, can be used. The redundancy can be of structural (space), temporal, or functional (informational) type. It is important that the quantity, the value of which is accepted as the reference one, depends on the main sources of the uncertainty growth to a significantly lesser extent than the measurand.

Taking into account the above-said, a concept of metrological self-check (MSC) was formulated. The MSC is an automatic check of metrological "health" of sensors and measuring systems [29. 30]. It enables detecting and evaluating deviations of the main metrological characteristics from their values stated at a previous calibration. Processing of MSC result sequences in the course of the operation process makes it possible to predict the time interval during which the metrological characteristics of each channel will correspond to those given in specifications.

In a number of cases, MSC enables correcting uncertainty or shifting to another operation algorithm to decrease this uncertainty. Such a way results in an opportunity to substantiate an increase in operation durability of a system measuring channel (without calibration or sensor replacement) in comparison with an analogous channel without the MSC function.

The development of the MSC theory, based on the redundancy application as well as documentary standards contributing to its implementation has been carried out in Russia since the early 80 s. In accordance with the Russian documentary standards [31, 32], measuring systems with MSC and sensors with MSC are called intelligent ones.

In various countries, teams dealing with the development of intelligent measuring systems and sensors were formed. For example, in Russia such teams were organized in Chelyabinsk, Ufa, Omsk, St. Petersburg, etc. Interesting works have been performed in Great Britain, Germany, USA, Ukraine, China, and other countries [17]. These approaches are applied in a number of sensors and measuring systems, e.g., described in [33-37].

Fig.1 illustrates the increase of interest, the specialists show in the development of intelligent measuring instruments corresponding to the above 
definition. The statistical data were obtained using the bibliographic database "Scopus". The vertical axis presents the percentage of publications in the field of "measurement", which contain the term "metrological self-check" or words close to it in meaning.

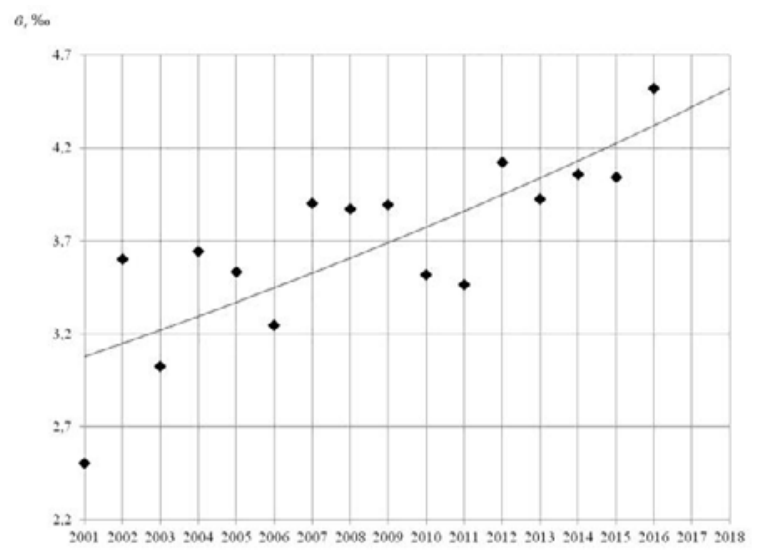

Fig.1. The level of interest the specialist show in the development of intelligent measuring instruments

Intelligent measuring system (IMS) can be built based on intelligent sensors included in measuring channels, but at present, a product range is very limited. However, IMS can be created also in the case if all the measuring channels contain sensors of conventional types (without any redundancy). Moreover, the MSC can reveal the growth of uncertainty caused by unexpected variation of measurement conditions.

In particular, MSC can be provided based on checking the correspondence between the sequences of results of measuring technological process parameters and patterns inherent in both the technological process and parameter interconnections. The MSC of such a type usually enables checking the level of critical uncertainty components, i.e., the components that are dominating or growing comparatively quickly.

To develop IMSs, at the starting stage, it is necessary to fulfill a metrological analysis of uncertainty components. It stipulates the studies of:

- $\quad$ expected operation conditions (including both a transient mode and steady run);

- operation experience of analogous sensors and measuring channels to estimate the character of uncertainty variations;

- "weak points" of analogs (design elements and units that are "responsible" for the uncertainty growth);

- features of design and manufacturing technology of measuring channels.

Results of additional tests (including forced tests) of analogs as well as units and prototypes of measuring channels under development can be supplemented to this list.

After such an analysis, uncertainty components should be ranked by the grade of danger in the course of longterm operation. Then, the critical uncertainty components should be chosen from them [29-32]. Developing CPSs, such a set of procedures is also necessary.

In a multichannel measuring system and, especially, in IMS or CPS, as a rule, a significant volume of calculations is carried out using measurement results characterized by uncertainties. Therefore, in the systems mentioned, realization of metrological auto-support of the metrological software is expedient. Such a support makes it possible to decrease the uncertainty and, as a result, to enhance the system metrological reliability too [38]. Besides, the metrological auto-support of software, which relies upon the patterns of information flow variations in a number of measuring channels and upon a time delay of these flows, should reveal and block hacker attacks.

\section{Measuring system with MSC as a model of CPS}

One of the multichannel systems that can be considered as a CPS simplified model is a system for measurement of pure water flow intended for water commercial accounting within a town or several its districts.

The channels with flowmeters are connected, practically, to the input of each house. A node flowmeter evaluates a total consumption of water in all apartments located in the house. Measurement information from each house is transmitted to a remote server of the measuring system, which calculates the cost of the water consumed.

The MSC can be organized using the comparison between information from flowmeters installed in apartments and the node one, analysis of flow dynamics as well as data obtained if the redundant but known information passes through a communication channel to the server. The MSC enables detecting flowmeters that need metrological maintenance, revealing sections of water supply requiring a priority checking, or, perhaps, immediate repairing as well as decreasing the risk of conflicts [37].

MSC of a system measuring tension of reinforcing steel ropes located on the concrete vessel of nuclear reactor, enhances the reliability of measurement information with regard to each rope. Thereby, taking into account climatic and other impacts, the MSC contributes to extension of safe operation lifetime of nuclear power plant.

As a simplest analogous system, it is possible to consider a system intended for providing diagnostics of threaded connections fastening a cover to a turbine unit body at a hydroelectric power plant. The thing is that a changing water pressure and vibration of the turbine body can weaken these connections. The early warning about a starting breakout process is the guarantee of safe operation.

IMS includes a set of 8 load washers (LW) mounted under internal screws on double-end bolts fastening the cover and an electronic unit connected with the load washers by high frequency cables [37, 39]. Each LW contains three measuring channels, each of them being made based on a quartz resonator. The LWs operate using surface acoustic waves. 
The detailed analysis has shown that MSC can be organized using the following "redundant" information:

- variations of a compression force for various LWs of this multichannelsystem, really, correlate with each other;

- in case of a defect in some measuring channel or in case of weakening of some threaded connection, variations of the values of the force measured, do not take place in all the LWs concurrently;

- a channel measuring the maximum compression force, is for the most part, the most stable; therefore, for each LW it can be used as a reference one.

Organization of the MSC enables enhancing a calibration interval for this system, and thereby, to decrease greatly the risk of accidents.

\section{Conclusion}

The era of cyber-physical systems and the Internet of Things is inevitably approaching, many people write about its attractive opportunities, but until present, there is still no unity in defining these terms.

The process of designing networks of interacting physical and computational components, which form the basis of "smart and safe cities", "smart healthcare" and other "smart" facilities, has already begun in a number of countries.

Nevertheless, attention to new metrological tasks related to ensuring the economic efficiency and safety of these networks is nit suficient.

In the networks, big volumes of information are generated, transmitted, received and processed. They are applied for making control decisions and actions as well. Traditional methods of metrological maintenance of measuring instruments in the course of their operation, stipulating 2- or 3-year intervals between metrological maintenance operations, are not economically acceptable for cyber-physical systems. At the same time, these methods unable providing the reliability of measurement information with the required probability.

It is necessary to develop international documentary standards, as well as methods and means (both hardware and software) focused on:

- $\quad$ improving the metrological reliability of CPSs components and organizing automatic continuous monitoring of the reliability of measurement information in cyber-physical systems and other IoT systems;

- $\quad$ protecting these systems from unauthorized access.

Methods of metrological self-check, which are developed for sensors and measuring systems of responsible designation, as well as national documentary standards in this field, should become, in our opinion, the basis of these developments.

\section{References}

1. Cyber-physical-systems [Internet]. Available: https://www.nist.gov/el/cyber-physical-systems.

2. F. Mattern, C. Floerkemeier, Lecture Notes in Comput. Sci., 6462, 242 (2010).
3. K. Lonergan, Taming the IoT terminology zoo: what does it all mean? [Internet]. Available: http://www.information-age.com/taming-iotterminology-zoo-what-does-it-all-mean$123459907 /$.

4. R. Minerva, A. Biru, D. Rotondi, Towards a definition of the Internet of Things (IoT) (Telecom Italia S.p.A., 2015).

5. V. Gunes, S. Peter, T. Givargis, F. Vahid, KSII T Internet Info, 8 (12), 4242 (2014)

6. R. Rajkumar, I. Lee, L. Sha, J. Stankovic, Proc. of 47th Design Autom. Conf., 731 (CA, USA, 2010).

7. Cyber-Physical Systems: Overview [Internet]. Available: http://www.sei.cmu.edu/cyber-physical/.

8. Ed. S. Yatsyshyn, B. Stadnyk, Cyber-Physical Systems: Metrological Issues (IFSA Publ., 2016).

9. L. Chernyak, Otkrytye Sistemy (Open Systems J), 2, 10 (2014). In Russian.

10. Recommend. ITU-T Y.2060: Overview of the Internet of Things (ITU-T, 2012).

11. Recommend. ITU-T Y.2069: Terms and definitions for the Internet of Things (ITU-T, 2012).

12. The Internet of Things: Opportunities and Applications across Industries (SAS, 2016).

13. EURAMET SRT-n02: Uniform communication interfaces in metrology for the Internet of Things (2015).

14. Enabling Consumer Connectivity Through Consensus Building [Internet]. Available: $\mathrm{http}: / /$ standardsinsight.com/ieee_company_detail/co nsensus-building.

15. IAB RFC 7452, Architectural Considerations in Smart Object Networking (2015).

16. The Internet of Things: Overview (Internet Society, 2015).

17. Internet of Things: Features, Challenges, and Vulnerabilities. [Internet]. Available:

http://elvedit.com/journals/IJACSIT/wpcontent/uploads/2015/02/internet-of-things.pdf

18. K. Sapozhnikova, A. Pronin, I. Druzhinin, R. Taymanov, Proc. of 14th IMEKO TC10 Workshop, 181 (Italy, 2016).

19. M. Chui, M. Loffler, R. Roberts, McKinsey Quaterly (March 2010).

20. Y. Sun, H. Song, A. J. Jara, R. Bie, IEEE Access: Spec. sect. on smart cities, 4, 766 (2016).

21. O. Vermesan, P. Friess, Internet of Things - From Research and Innovation to Market Deployment (River Publish., Denmark, 2014). 
22. Z. Javeed, Sensors, Environment and Internet of Things (IoT) [Internet]. Available: https:/www.talend.com/blog/2016/12/08/sensorsenvironment-and-internet-of-things-iot/.

23. Recommend. RMG 29-2013: Metrology. Basic terms and definitions (Moscow, Russia, 2014).

24. H.M. Hashemian, Sensor Performance and Reliability (ISA, USA, 2005).

25. Y.-K. Chen, 17th ASP-DAC, 383 (IEEE, Australia, 2012).

26. J. Gubbi, R. Buyya, S. Marusic, M. Palaniswami, Future Gener. Comput. Syst., 29 (7), 1645 (2013).

27. GOST R 8.825-2013: Intelligent Sensors and Intelligent Measuring Systems. Methods of accelerated tests (Moscow, 2014). In Russian.

28. D. Galar, Proc. of 14th IMEKO TC10 Workshop, 8 (Italy, 2016).

29. R. Taymanov, K. Sapozhnikova, Measurement 43 (7), 869 (2010).

30. R. Taymanov, K. Sapozhnikova, Ch. 1 in: S. Nihtianov, A. L. Estepa, Smart Sensors and MEMS for Industrial Applications (Woodhead Publ. Ltd., 2014), 3.
31. GOST R 8.673-2009: Intelligent Sensors and Intelligent Measuring Systems. Basic Terms and Definitions (Moscow, 2010). In Russian.

32. GOST R 8.734-2011: Intelligent Sensors and Intelligent Measuring Systems. Methods of Metrological Self-checking (Moscow, 2011). In Russian.

33. A. Ionov, B. Ionov, N. Chernysheva, E. Plotkin, Key Eng. Mater., 613, 307 (2014).

34. V. Gurevich, K. Sapozhnikova, R. Taymanov, Proc of 10th ISMTII, D5-4, (S. Korea, 2011).

35. T. Watanabe, Proc. of 11th ISMTII (Germany, 2013).

36. R. Taymanov, K. Sapozhnikova, I. Druzhinin, Sensors\&Transducers J., 10 (2), 30 (2011).

37. R. Taymanov, K. Sapozhnikova, I. Danilova, I. Druzhinin, Proc. of XXI IMEKO WC (Czech Rep., 2015).

38. K. K. Semenov, G. N. Solopchenko, Proc. of XXI IMEKO WC, 2035 (Czech Rep., 2015).

39. K. Sapozhnikova, R. Taymanov, I. Danilova, I. Druzhinin, Proc. of 12th ISMTII, 59 (Taiwan, 2015). 